\title{
On the Infrared Behavior of Green's Functions in Yang-Mills Theory
}

\author{
Axel Maas, Attilio Cucchieri, and Tereza Mendes \\ Instituto de Física de São Carlos, Universidade de São Paulo, \\ Caixa Postal 369, 13560-970 São Carlos, SP, Brazil
}

(Received on 27 September, 2006)

\begin{abstract}
Non-perturbative properties of QCD, such as color confinement, are encoded in the infrared behavior of correlation functions, e.g. propagators and vertices. Various analytic predictions have been suggested for these quantities in various gauges. Here we numerically test these predictions using lattice gauge theory. In particular, we present results for the 2- and 3-point functions for $S U(2)$ Landau-gauge Yang-Mills theory in three and in four dimensions. Special attention is paid to systematic finite-volume effects. The gluon and ghost propagators are also evaluated in the so-called interpolating gauge (between the Landau and the Coulomb gauge), in order to study their gauge-dependence. Finally, we consider these propagators in Landau gauge at finite temperature, with the aim of understanding the effect of the deconfinement phase transition on their infrared behavior. All our results are compatible with the so-called Gribov-Zwanziger confinement scenario.
\end{abstract}

Keywords: Yang-Mills theory; Confinement; Propagators; Vertices; Landau gauge; Interpolating gauge; Finite temperature

\section{INTRODUCTION}

The confinement phenomenon in QCD is a long-range effect and one can expect its explanation to be encoded in the infrared (IR) behavior of the QCD correlation functions. Indeed, various confinement scenarios make predictions for this behavior [1]. Unfortunately, a quantitative determination of these correlation functions is a very difficult task.

Continuum methods, based e.g. on Dyson-Schwinger equations (DSEs) [1-4], on the renormalization group [5] or on effective potentials [6], lead to results that seem to agree with the Gribov-Zwanziger confinement scenario [4, 7] and with the Kugo-Ojima scenario [8]. These methods have the disadvantage of introducing approximations, which in general cannot be controlled and verified. On the other hand, numerical studies using lattice gauge theory allow a determination of QCD correlation functions in the IR limit using approximations that can in principle be controlled and quantified $[9,10]$. In this case, since one has to work with finite volumes $N^{d}$, the system considered has a natural IR cutoff $p \sim 1 /(a N)$, making difficult the study of the true IR limit. (This topic will be discussed in more detail in Section II.) Clearly, a close cooperation between continuum and lattice methods can be a promising approach. In particular, lattice gauge theory allows to check the approximations usually considered in continuum methods, or at least to provide constraints for possible Ansätze. The most important test is probably the verification of the approximations employed for the 3-point vertices when solving DSEs. Results for the three-gluon and the ghost-gluon vertices will be reported in Section III.

Most of the studies of QCD correlations functions, both in the continuum and on the lattice, have been done in Landau gauge. On the other hand, since these quantities are gauge dependent, it is interesting to evaluate them in other gauges too (e.g. Coulomb gauge [11] and maximally Abelian gauge [12]). In principle, the explanation of the confinement mechanism based on the IR properties of the QCD correlation function can also be gauge-dependent. Particularly important are the so-called interpolating gauges $[13,14]$, since they allow to relate different gauges and possible different confinement mechanisms. In Section IV we report $3 d$ results for the gauge interpolating between the Landau and the Coulomb gauge.

Finally, a clear understanding of the confinement mechanism could be useful in the study of the QCD phase diagram and of the deconfining phase transition [15]. In particular, one might expect to observe a modification of the IR properties of the correlation functions at finite temperature $T$. As a first step in this direction, the gluon and ghost propagators at finite $T$ are considered (in the $4 d$ Landau gauge) in Section V.

\section{FINITE-VOLUME EFFECTS}

Confinement is induced by long-range correlations. More precisely, the correlation length should be infinite [1]. Thus, when considering QCD correlation functions in momentum space, confinement should be related to their IR properties. This is the case for the Gribov-Zwanziger and the KugoOjima confinement scenarios. Indeed, they predict a specific IR behavior for the gluon and the ghost propagators in Landau gauge: the gluon propagator should be IR suppressed - and it is expected to vanish at zero momentum - while the ghost propagator should be IR enhanced compared to the one of a massless particle.

As said above, the IR-cutoff $1 /(a N)$ makes it difficult to study the IR properties of the correlation function and one should pay attention to possible finite-size effects. Of course, this analysis can be computationally very demanding. In this case, the consideration of three-dimensional lattices can be useful in order to access large lattice volumes. Let us note that the Gribov-Zwanziger scenario applies also to the threedimensional case [4] and that results for the IR behavior of the propagators using DSEs are available for $d=3[4,16]$.

In Figure 1 we report the comparison of DSE results with lattice data for the gluon and the ghost propagators. In the first case, finite-volume effects are evident for the smallest momenta. In particular, as the volume increases, the propagator becomes more and more IR suppressed. At the same time, the difference between two different lattices with a given volume ratio moves further and further into the IR limit with increas- 


\section{Gluon propagator}

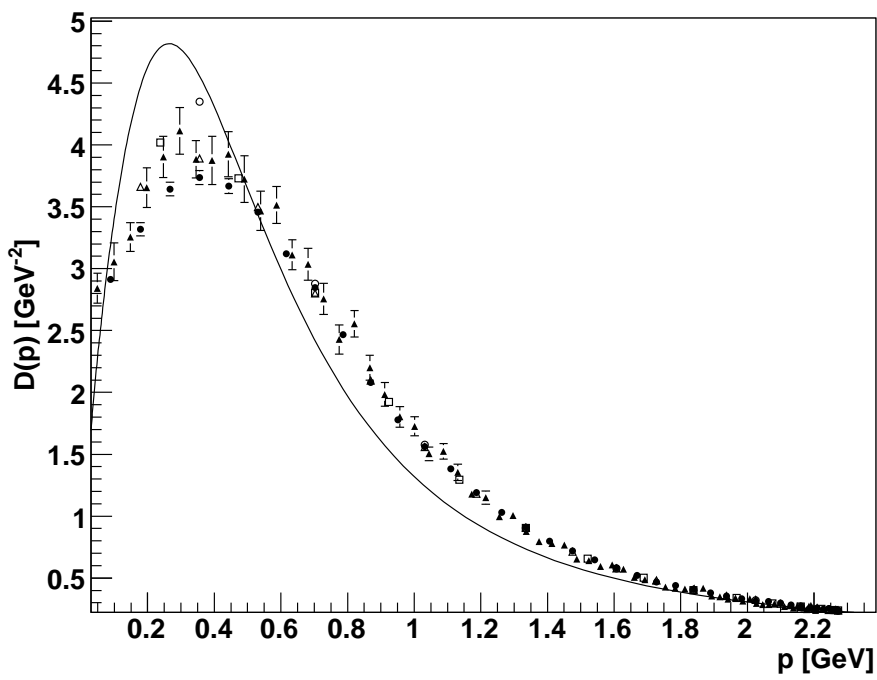

\section{Ghost propagator}

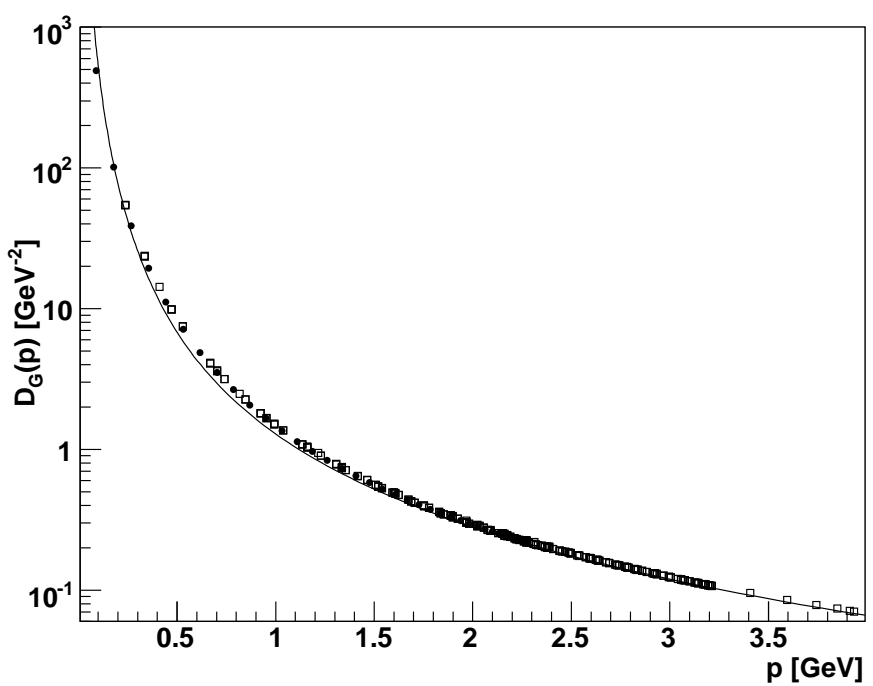

FIG. 1: Comparison of lattice data and DSE results for gluon $D(p)$ (left) and ghost $D_{G}(p)$ (right) propagators in the $3 d$ case in Landau gauge as a function of the momentum $p$. The solid line is from DSE results [16]. Lattice simulations have been done at $\beta=4.2$ considering various lattice volumes. Open circles correspond to lattice volume $20^{3}\left[V \approx(3.5 \mathrm{fm})^{3}\right]$ [18], open squares represent $30^{3}\left[V \approx(5.2 \mathrm{fm})^{3}\right][18]$, open triangles are used for $40^{3}\left[V \approx(6.9 \mathrm{fm})^{3}\right]$ [19], full circles correspond to $80^{3}\left[V \approx(14 \mathrm{fm})^{3}\right]$ [20] and full triangles represent lattice volume $140^{3}\left[V \approx(24 \mathrm{fm})^{3}\right][21]$.

ing volume. Thus, momenta of the order of $2 \sin (\pi / N) / a \approx$ $2 \pi /(a N)$ are affected by finite-volume effects. From Figure 1 one also sees that DSE and lattice results are in qualitatively agreement. Let us remark that DSE studies predict [17] that the true IR regime is reached only below a certain momentum scale $\Lambda_{I} \lesssim 200 \mathrm{MeV}$, i.e. on a finite volume one should have a sufficient number of non-zero momenta in the range $2 \pi /(a N) \ll p \lesssim \Lambda_{I}$. With a lattice spacing $a$ of order of 1 $\mathrm{GeV}^{-1}$ one needs $N \gg 50$. Unfortunately, this regime is just reached by the lattices in the $3 d$ case but not yet in $4 d$.

The ghost propagator is much less affected by finite-volume effects, even though small effects can be found for the dressing function at small momenta [19]. Moreover, the comparison of the lattice data to DSE results is quite good (see Fig. 1). One should however recall that the IR enhancement of the ghost propagator seems to be related to an appropriate sampling of the so-called exceptional configurations $[10,18]$. Thus, results obtained considering a relatively small statistics are likely underestimating this IR enhancement.

We conclude that the smallest non-zero lattice momenta of the order of $2 \pi /(a N)$ - are probably not reliable in lattice studies and should be treated with great care. A more thorough discussion of finite-size effects will be presented in [19].

\section{THREE-POINT VERTICES}

Besides the propagators, also the vertices are of great importance in understanding the IR properties of QCD. In particular, assumptions on their IR behavior are crucial inputs for the analytic methods [1-3]. At the same time, predictions for their IR behavior have been obtained using continuum methods [22-24]. As stressed in the Introduction, lattice gauge theory provides us with the possibility of verifying these results as well as the assumptions considered in analytic studies. The simplest of these vertices are the 3-point ones. In Landau-gauge pure Yang-Mills theory one can consider the ghost-gluon vertex and the three-gluon vertex. Kinematically these vertices are much more complicated than the propagators, since they depend on three independent kinematic variables. In addition, they have a complex tensor structure (especially the three-gluon vertex).

In order to simplify the presentation, we consider here only one specific kinematic configuration, namely the one in which two of the incoming momenta are orthogonal with respect to each other (see $[18,25]$ for details and for other possible kinematic configurations). Furthermore, instead of the various tensor elements we evaluate the quantity [18]

$$
G=\frac{\Gamma^{\mathrm{tl}, a b c} G^{a b c}}{\Gamma^{\mathrm{t} l a b c} D^{a d} D^{b e} D^{c f} \Gamma^{\mathrm{tl}, d e f}} .
$$

Here the indices $a, \ldots, f$ are generic multi-indices, encompassing field-type, Lorentz and color indices. Also, $D^{a b}$ are the propagators of the fields, $G^{a b c}$ represent the full Green's functions and $\Gamma^{\mathrm{t} l, a b c}$ are the corresponding tree-level vertices. This quantity is defined such that it becomes equal to one if the full and the tree-level vertex coincide. Furthermore, the ratio (1) corresponds to the kernel of a one-loop not-amputated self-energy diagram in DSEs over the same kernel with full vertices replaced by the bare ones. Thus, the quantity $G$ is a direct measure of how good the approximations in DSE calculations are when the bare vertices are used. 


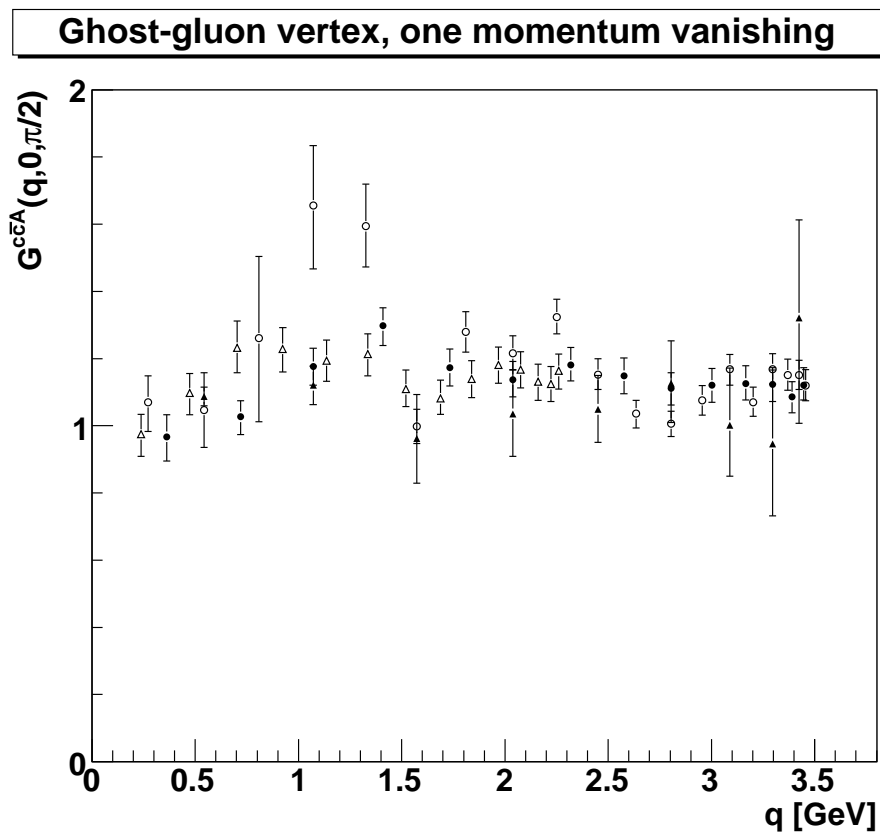

Ghost-gluon vertex, orthogonal momenta

\section{Three-gluon vertex, one momentum vanishing}
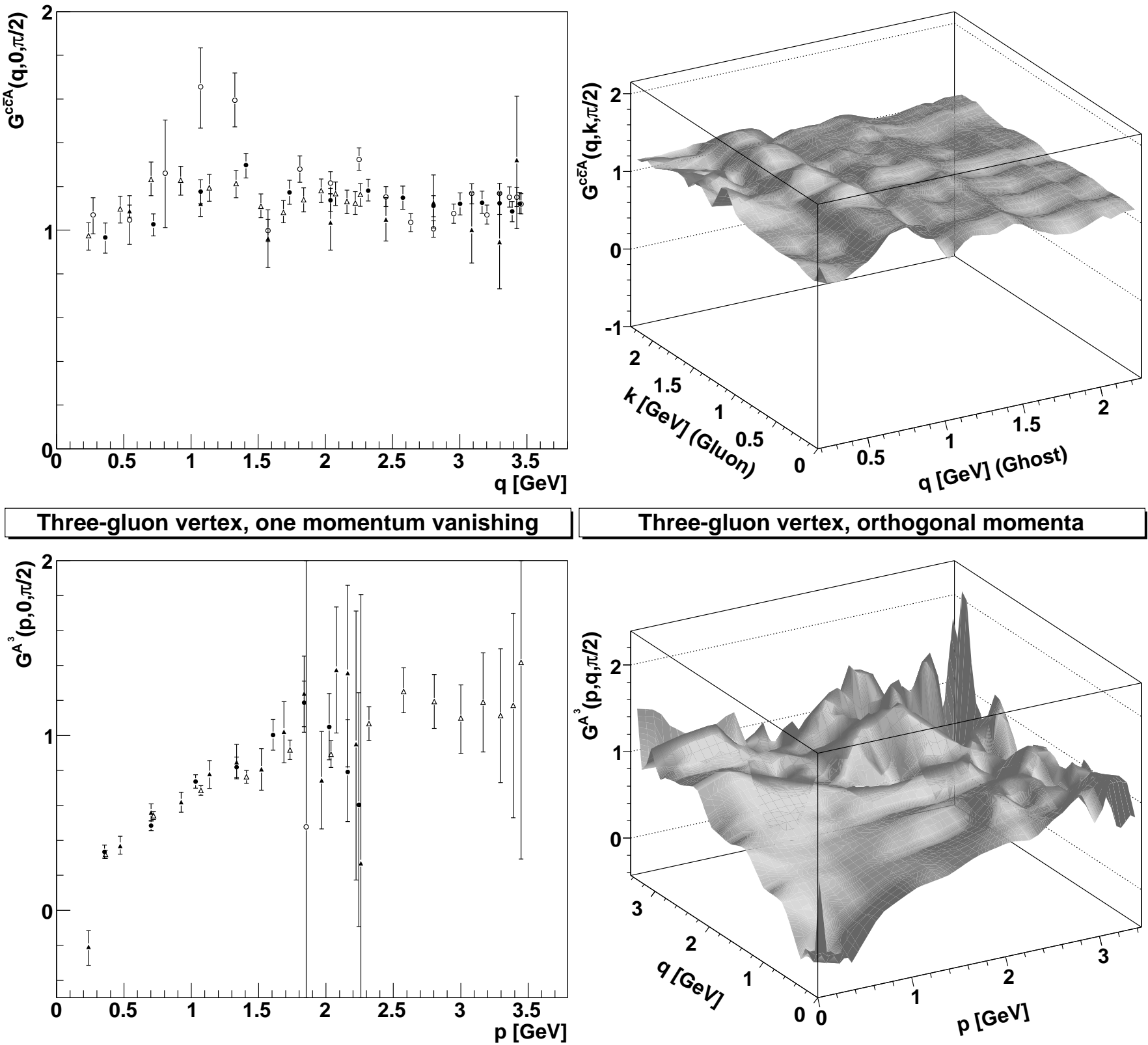

FIG. 2: Top-left panel: ghost-gluon vertex at zero gluon momentum; open triangles are used for $V=30^{3}$ [25] and open circles for $V=30^{3}$ (in both cases $\beta=4.2$ ), full triangles are used for $V=20^{3}$ [18] and full circles for $V=30^{3}$ (in both cases $\beta=6.0$ ). Top-right panel: ghost-gluon vertex with the gluon and the ghost momenta orthogonal to each other; the graph interpolates data obtained from a $40^{3}$ lattice at $\beta=4.2$ [25]. The spike at zero momentum is an artifact, as the vertex function cannot be evaluated there. Bottom-left panel: 3-gluon vertex with one external momentum vanishing [18]; full symbols correspond to $\beta=4.2$ and open symbols to $\beta=6.0$; circles are used for $V=20^{3}$ and triangles for $V=30^{3}$. Bottom-right panel: 3-gluon vertex with two external momenta orthogonal to each other [18]; the graph interpolates data obtained from a $30^{3}$ lattice at $\beta=4.2$ and at $\beta=6.0$. Note that the spike at zero momentum is an artifact of the interpolating algorithm, since the vertex cannot be evaluated there.

Results for the quantity (1) in three and in four dimensions for both vertices are shown in Figures 2 and 3, respectively. The ghost-gluon vertex is essentially constant for all momenta studied here as well as for other kinematic configurations [18, $25,26]$, both in $3 d$ and in $4 d$. In particular, there is no sign of enhancement or suppression even for the smallest momenta available. This is in agreement with results obtained using continuum methods [23,24]. In addition, the constancy of this vertex confirms the most important assumption made in DSE studies, i.e. the use of a bare ghost-gluon vertex $[1,2,16,24]$. 


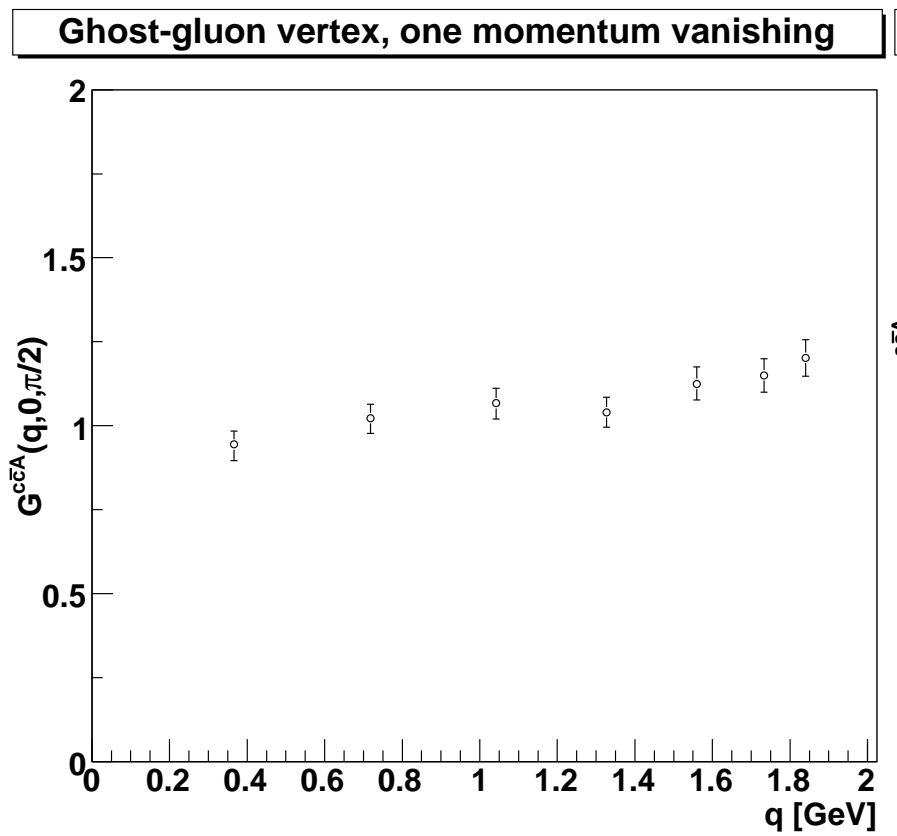

\section{Ghost-gluon vertex, orthogonal momenta}

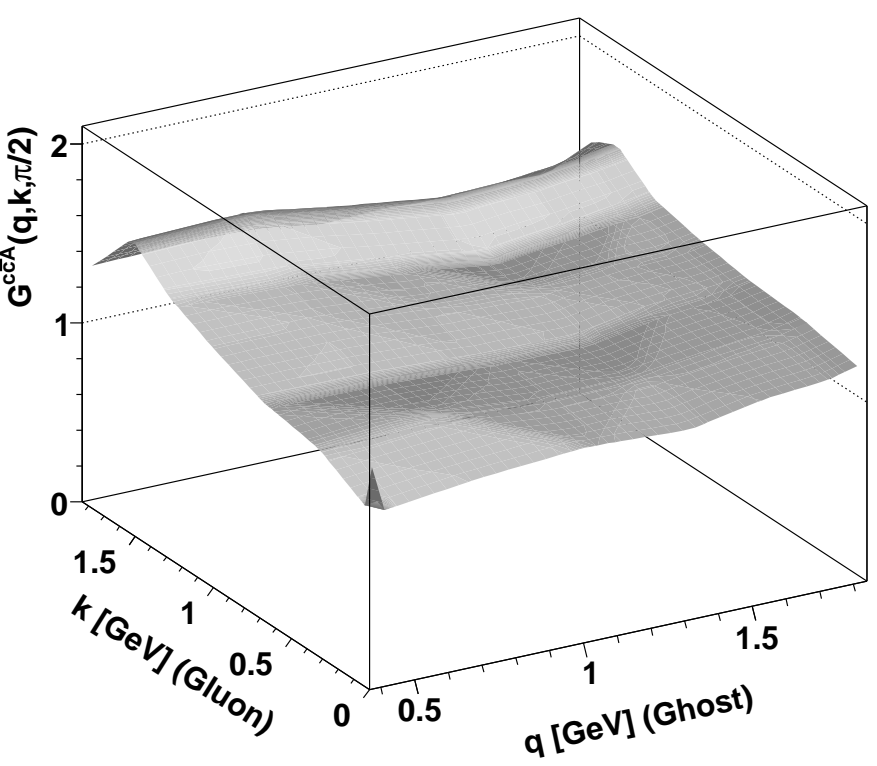

Three-gluon vertex, one momentum vanishing

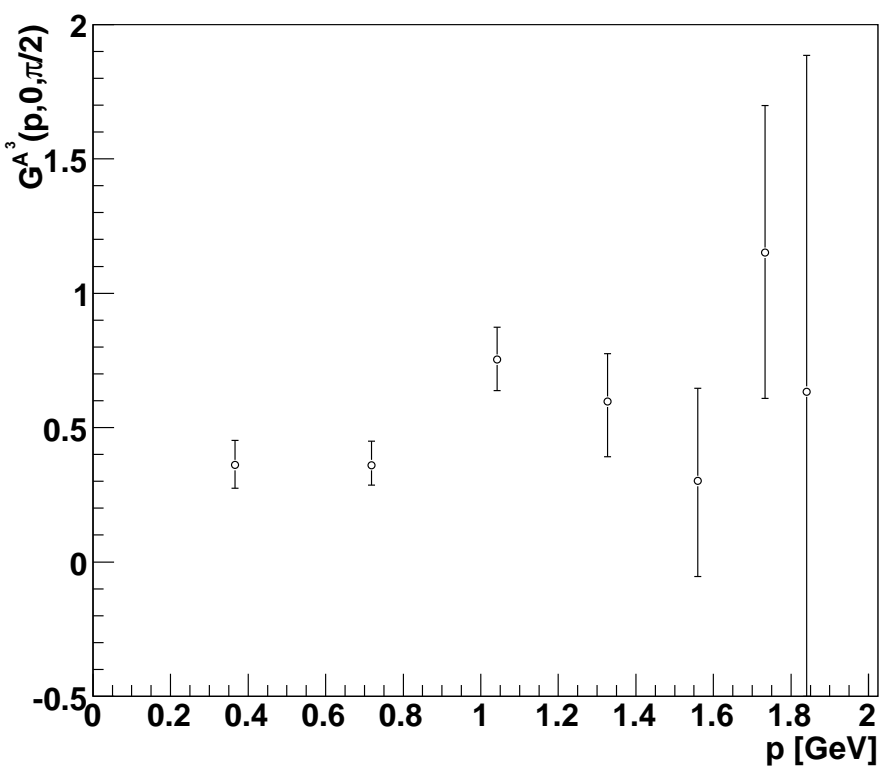

\section{Three-gluon vertex, orthogonal momenta}

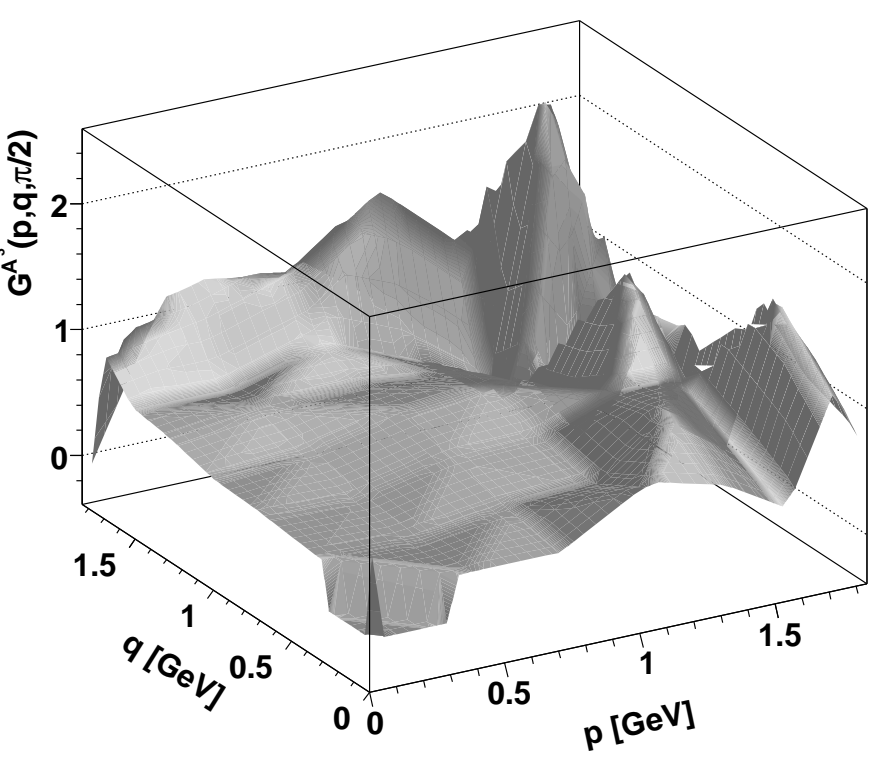

FIG. 3: Same as Figure 2 but in the four-dimensional case. Here the lattice volume is $16^{4}$ and simulations have been done at $\beta=2.2$ [25].

The evaluation of three-gluon vertex is numerically much more demanding than that of the ghost-gluon vertex. In particular, very large statistics are required for its investigation $[18,25]$, especially in the three-dimensional case. Our results show that this vertex is suppressed at intermediate momenta, especially for $d=3$. On the other hand, with our data, it is difficult to understand completely its precise IR behavior. Let us note that, if this suppression were to be confirmed by studies using larger lattices, this result would contradict predictions obtained from DSE studies [22, 24]. Nevertheless, it would not invalidate the assumptions usually made in the continuum calculations of the propagators $[1,2,16,24]$.

\section{INTERPOLATING GAUGES}

All results presented so far have been obtained in the (minimal) Landau gauge. As said in the Introduction, it is eventually desirable to understand confinement in a gaugeindependent way, if this is possible. Thus, the IR properties of the QCD correlation functions in other gauges are also interesting. Among other possible gauge-fixing conditions, the 


\section{Spatial gluon propagator $D^{\text {tr }}$}

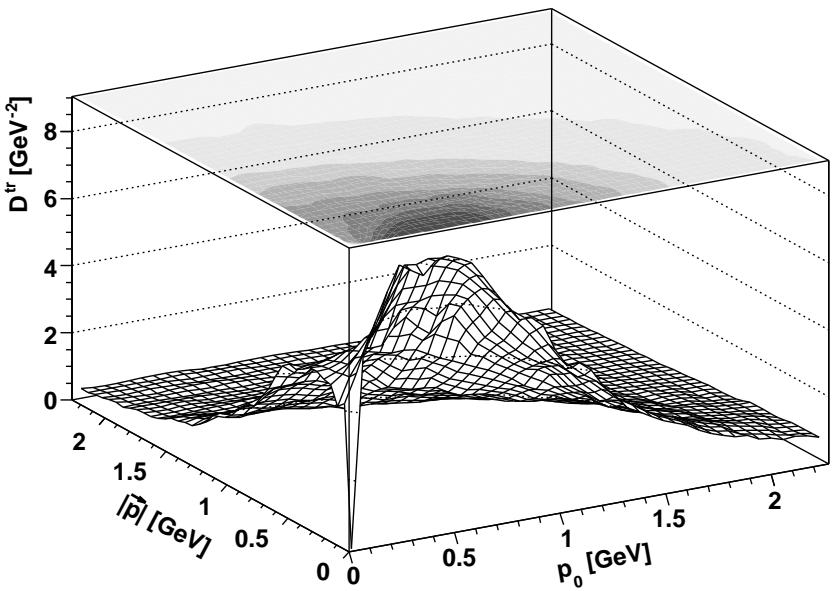

Temporal gluon propagator $\mathbf{D}_{00}$

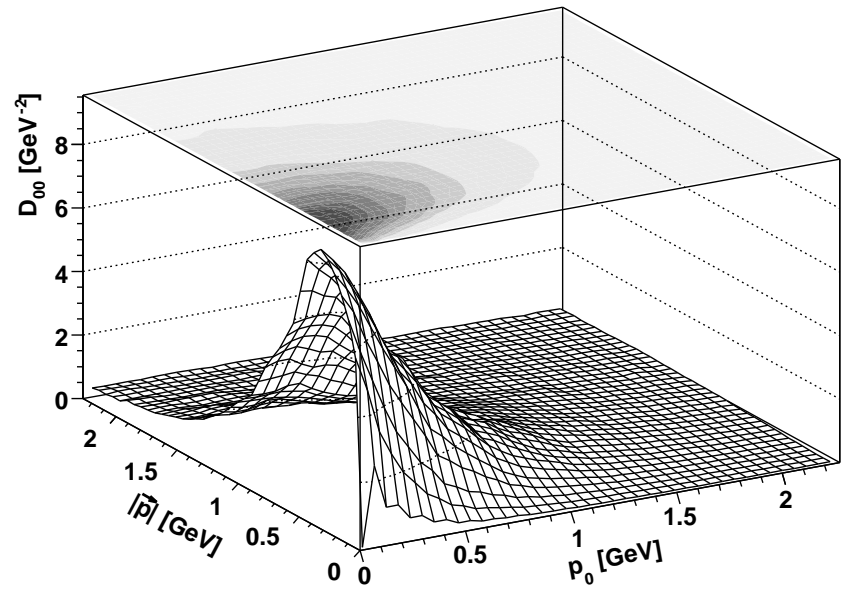

FIG. 4: The spatial (top) and temporal (bottom) gluon propagator in the interpolating gauge (2) at $\lambda=1 / 2$ as a function of the temporal $p_{0}$ and spatial $|\vec{p}|$ momenta, from a $60^{3}$ lattice at $\beta=4.2$ [29]. Results have been interpolated; the spike at zero is an artifact of the missing point there.

so-called interpolating gauges allow to interpolate smoothly between specific gauge conditions. One example is given by the gauge condition $[13,27]$

$$
\lambda \partial_{0} A_{0}^{b}+\vec{\partial} \vec{A}^{b}=0 .
$$

This gauge interpolates between the Landau gauge $(\lambda=1)$ and the Coulomb gauge $(\lambda=0)$, even though the Coulomb gauge limit itself is likely not to be a smooth one [28]. Nonetheless, this gauge allows one to study the Gribov-Zwanziger scenario when moving away from the Landau gauge. Since for $\lambda \neq 1$ the $O(4)$-invariance is explicitly broken, it is natural to consider two scalar gluon propagators [28], i.e. the timetime component $D_{00}^{a a} /\left(N_{c}^{2}-1\right)$ and the 3-dimensional transverse one $D^{\mathrm{tr}}=D_{i i}^{a a} /\left((d-1)\left(N_{c}^{2}-1\right)\right)$. In addition, the propagators depend independently on the temporal momentum $p_{0}$ and on the spatial momentum $|\vec{p}|$.

\section{Ghost dressing function}

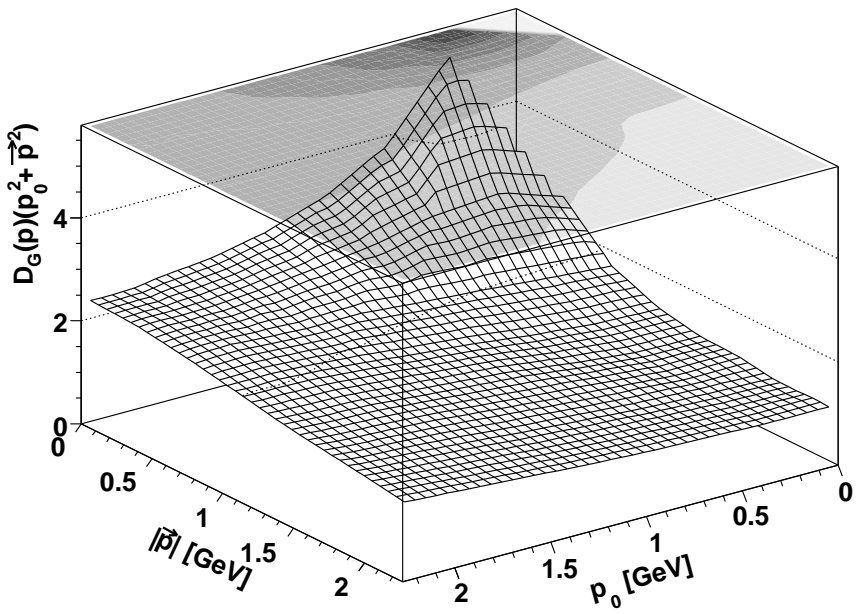

FIG. 5: The ghost dressing function $D_{G}(p)\left(p_{0}^{2}+\vec{p}^{2}\right)$ in the interpolating gauge (2) at $\lambda=1 / 2$ as a function of the temporal $p_{0}$ and spatial $|\vec{p}|$ momenta, from a $20^{3}$ lattice at $\beta=4.2$ [29]. Numerical data have been interpolated.

Results at $\lambda=1 / 2$ are shown in Figure 4 for these two gluon propagators and in Figure 5 for the scalar ghost dressing function $D_{G}(p)\left(p_{0}^{2}+\vec{p}^{2}\right)$. Note that $D_{00}\left(p_{0}, 0\right)$ vanishes exactly for any $p_{0}$ due to the gauge condition, even though (due to technical reasons) this is not clear from Figure 4. Both gluon propagators are found to be IR suppressed when considered as a function of $p_{0}^{2}+\vec{p}^{2}$. Also, both propagators exhibit a clear maximum. Furthermore, both propagators are not suppressed as long as either $p_{0}$ or $|\vec{p}|$ are not sufficiently small. At the same time, the ghost propagator is IR enhanced. The difference in behavior at large pure-temporal and at large pure-spatial momenta is a direct consequence of how $\lambda$ appears in the tree-level propagators. Thus, the IR behavior of these propagators is (qualitatively) the same as that found in Landau gauge. This is in agreement with results from DSE studies [28]. On the other hand, since the limit $\lambda \rightarrow 1$ is smooth, one wouldn't expect a strong deviation from the Landau behavior for a value $\lambda=1 / 2$. Results for the non-smooth limit $\lambda \rightarrow 0$ will be presented elsewhere [29].

\section{FINITE TEMPERATURE}

Yang-Mills theory undergoes a deconfining phase transition at finite temperature [15] and one expects this transition to change the confining properties of the theory. At the same time, if confinement is manifest in the IR properties of the QCD propagators, these might also change across the phase transition. At finite temperature, the gluon propagator can no longer be described by a single tensor structure and one needs two independent structures [30]:

$$
D_{\mu \nu}^{a b}=P_{\mu v}^{T} D^{T a b}+P_{\mu \nu}^{L} D^{L a b} .
$$




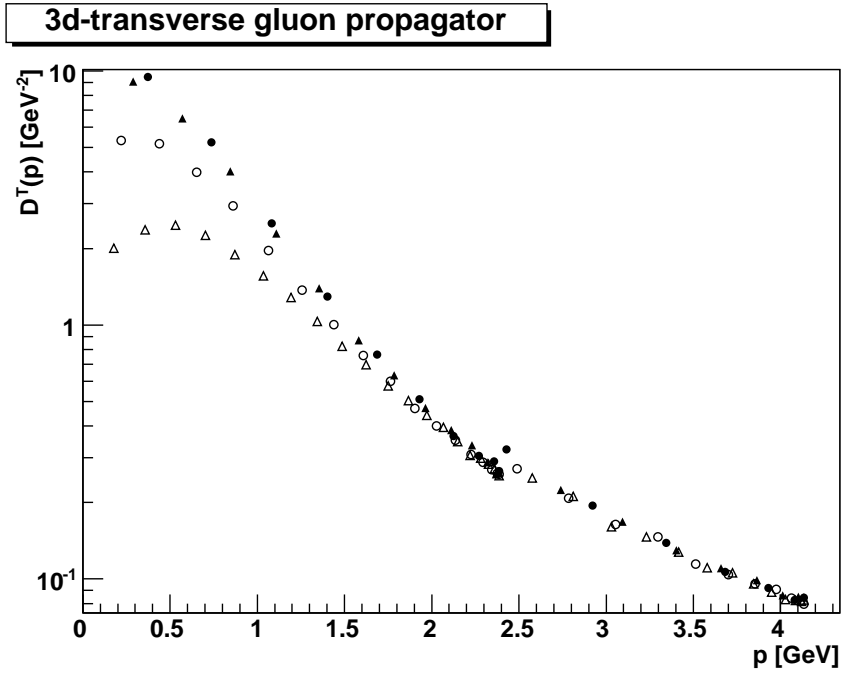

3d-longitudinal gluon propagator

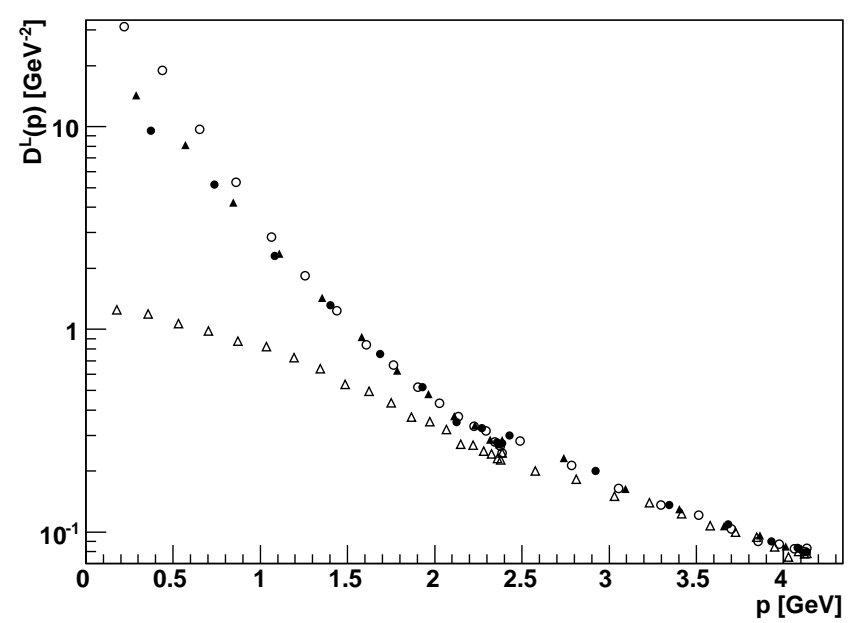

FIG. 6: The spatially transverse (top) and spatially longitudinal (bottom) gluon propagators at various temperatures [19]. Full circles are at $T=0$ on a $20^{4}$ lattice, full triangles are at $T \approx 149 \mathrm{MeV}$ on a $10 \times 26^{3}$ lattice, open circles are at $T \approx 298 \mathrm{MeV}$ on a $4 \times 34^{3}$ lattice, and open triangles are at $T \approx 597 \mathrm{MeV}$ on a $2 \times 42^{3}$ lattice. All results have been obtained using $\beta=2.3$.

Here, $P_{\mu \nu}^{T}$ is purely spatial and transverse while $P_{\mu \nu}^{L}=P_{\mu \nu}-$ $P_{\mu \nu}^{T}$, i.e. $P_{\mu \nu}^{L}$ complements $P_{\mu \nu}^{T}$ to a four-dimensional-transverse $O(4)$-invariant tensor-structure $P_{\mu \nu}=\delta_{\mu \nu}-p_{\mu} p_{v} / p^{2}$. In addition, $P_{\mu v}^{L}$ is longitudinal in the spatial sub-space. Furthermore, all functions depend on the spatial momentum $|\vec{p}|$ and on the energy $p_{0}$ separately. The latter is discrete with values $p_{0}=2 \pi T n$, where $n$ is an integer. Infrared or soft modes are those with zero energy, i.e. $n=0$.

Results for the soft modes of the two gluon propagators and of the (scalar) ghost propagator are shown in Figures 6 and 7 for temperatures above and below the phase transition. Clearly, as expected, all propagators coincide with the zerotemperature perturbative tail for momenta $p$ large compared to the temperature $T$. At the same time, each of the three

\section{Ghost propagator}

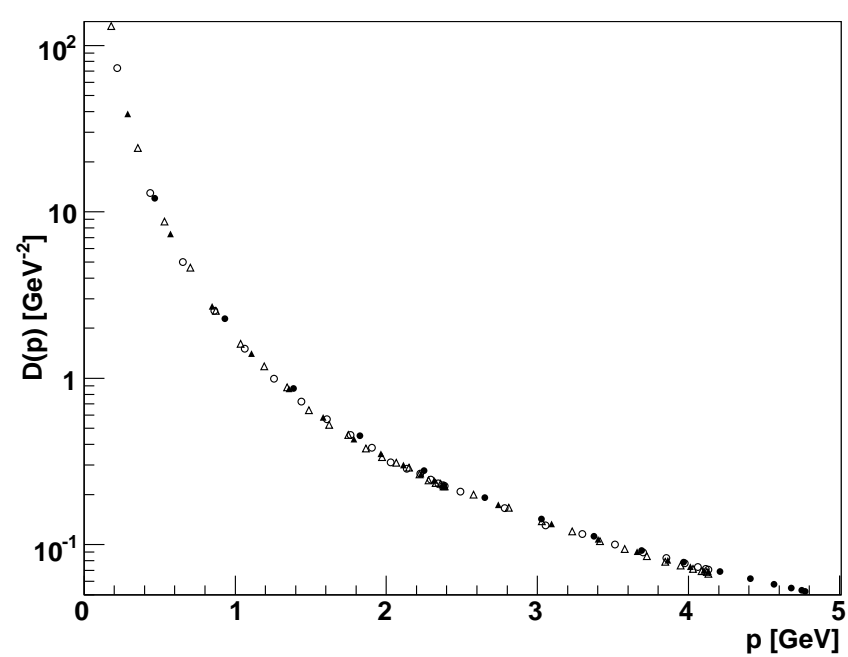

FIG. 7: Same as figure 6, but for the ghost propagator. Also the symbols are the same except for full circles, which refer to a $32^{4}$ lattice at $\beta=2.3(T=0)$.

propagators shows a different temperature dependence at low momenta. In particular, the $3 d$-transverse propagator $D^{T}(p)$ decreases smoothly (in the IR) with increasing temperature. At the same time, for the highest temperature considered here $(T \approx 597 \mathrm{MeV})$, one sees a clear IR suppression with a maximum for $p \approx 600 \mathrm{MeV}$. Let us stress that, while at $T=0$ one does not see a decreasing propagator $D^{T}(p)$ even for a lattice volume of $52^{4}$ [31], for this temperature above the phase transition the IR suppression is already manifest for a spatial volume of $42^{3}$. This suggests that, at high temperature, either the infrared suppression is stronger or that finite-volume effects are smaller, compared to the zero-temperature case. In the case of the $3 d$-longitudinal propagator $D^{L}(p)$ we observe an increase as the temperature is turned on starting from $T=0$. However, the propagator decreases (in the IR region) when going from the second-highest to the highest temperature. A possibility would be a propagator increasing (respectively decreasing) when the temperature rises for temperatures below (respectively above) the phase transition. Let us note that $D^{L}(p)$ does not seem to be IR suppressed, even though in the IR limit it does not grow as strongly as the propagator of a massless particle [19]. Finally, the ghost propagator does not show any visible temperature dependence. Let us also stress that these results should be taken with care, especially when considering the $3 d$-longitudinal gluon propagator $D^{L}(p)$, since we find strong volume and discretization effects[19].

These results are in agreement with previous lattice [32] and continuum results [33] and they can be interpreted considering a Gribov-Zwanziger-type scenario [19]. In particular, they suggest that this confinement scenario prevails at all temperatures, i.e. not only for small temperature, but also in the high-temperature phase. 


\section{SUMMARY}

Summarizing, we have presented results for gluon and ghost propagators considering the 3 - and the 4-dimensional cases for various gauges at zero temperature. In all cases our results are in qualitatively agreement with the Kugo-Ojima and with the Gribov-Zwanziger scenarios. Moreover, an investigation of the propagators in the interpolating gauge (2) suggests that the Gribov-Zwanziger scenario is stable when moving away from the Landau gauge condition.

At zero temperature, in Landau gauge, we have also evaluated the ghost-gluon and the 3-gluon vertices. This study provides additional hints and constraints for the assumptions usually considered in continuum studies.

Finally, we have presented results for the two propagators at finite temperature around the deconfining phase transition.
These results fit into an extension of the Gribov-Zwanziger scenario at finite temperature $[19,32,33]$.

In all cases considered, the analysis of finite-volume effects should be done very carefully, especially for the gluon propagator. Thus, a complete understanding of the IR behavior of the QCD correlation functions and a confirmation (or disproof) of the available confinement scenarios will be obtained only when extending these studies to larger lattices.

\section{Acknowledgments}

A. M. was supported by the DFG under grant number MA 3935/1-1. A. C. and T. M. were supported by FAPESP (under grant \# 00/ 05047-5) and by CNPq.
[1] R. Alkofer and L. von Smekal, Phys. Rept. 353, 281 (2001), and references therein.

[2] P. Watson and R. Alkofer, Phys. Rev. Lett. 86, 5239 (2001); C. Lerche and L. von Smekal, Phys. Rev. D 65, 125006 (2002); C. S. Fischer, J. Phys. G 32, R253 (2006), and references therein;

[3] L. von Smekal, R. Alkofer, and A. Hauck, Phys. Rev. Lett. 79, 3591 (1997); Annals Phys. 267, 1 (1998) [Erratum-ibid. 269, 182 (1998)]; C. S. Fischer and R. Alkofer, Phys. Lett. B 536, 177 (2002); C. S. Fischer, R. Alkofer, and H. Reinhardt, Phys. Rev. D 65, 094008 (2002).

[4] D. Zwanziger, Phys. Rev. D 65, 094039 (2002); Phys. Rev. D 69, 016002 (2004), and references therein.

[5] H. Gies, Phys. Rev. D 66, 025006 (2002); C. S. Fischer and H. Gies, JHEP 0410, 048 (2004); J. M. Pawlowski, D. F. Litim, S. Nedelko, and L. von Smekal, Phys. Rev. Lett. 93, 152002 (2004).

[6] D. Dudal, R. F. Sobreiro, S. P. Sorella, and H. Verschelde, Phys. Rev. D 72, 014016 (2005).

[7] V. N. Gribov, Nucl. Phys. B 139, 1 (1978); D. Zwanziger, Nucl. Phys. B 412, 657 (1994); Phys. Rev. D 67, 105001 (2003).

[8] T. Kugo and I. Ojima, Prog. Theor. Phys. Suppl. 66, 1 (1979) [Erratum Prog. Theor. Phys. 71, 1121 (1984)]; T. Kugo, hepth/9511033.

[9] J. C. R. Bloch, A. Cucchieri, K. Langfeld, and T. Mendes, Nucl. Phys. B 687, 76 (2004); P. O. Bowman, U. M. Heller, D. B. Leinweber, M. B. Parappilly, and A. G. Williams, Phys. Rev. D 70, 034509 (2004)

[10] A. Sternbeck, E. M. Ilgenfritz, M. Müller-Preussker, and A. Schiller, Phys. Rev. D 72, 014507 (2005).

[11] H. Reinhardt and C. Feuchter, Phys. Rev. D 71, 105002 (2005); C. Feuchter and H. Reinhardt, Phys. Rev. D 70, 105021 (2004); A. Cucchieri and D. Zwanziger, Phys. Rev. D 65, 014001 (2002).

[12] V. G. Bornyakov, M. N. Chernodub, F. V. Gubarev, S. M. Morozov, and M. I. Polikarpov, Phys. Lett. B 559, 214 (2003); M. A. L. Capri et al., hep-th/0603167; A. Cucchieri, A. Mihara, and T. Mendes, in preparation.

[13] L. Baulieu and D. Zwanziger, Nucl. Phys. B 548, 527 (1999).
[14] M. A. L. Capri, R. F. Sobreiro, and S. P. Sorella, Phys. Rev. D 73, 041701 (2006).

[15] F. Karsch, Lect. Notes Phys. 583, 209 (2002), and references therein.

[16] A. Maas, J. Wambach, B. Grüter, and R. Alkofer, Eur. Phys. J. C 37, 335 (2004).

[17] C. S. Fischer, A. Maas, J. M. Pawlowski, L. von Smekal,

[18] A. Cucchieri, A. Maas, and T. Mendes, Phys. Rev. D 74, 014503 (2006).

[19] A. Cucchieri, A. Maas, and T. Mendes, is just available as [arXiv:hep-ph/0701050], and not yet published.

[20] A. Cucchieri and T. Mendes, Phys. Rev. D 73, 071502 (2006).

[21] A. Cucchieri, T. Mendes, and A. R. Taurines, Phys. Rev. D 67, 091502 (2003).

[22] W. Schleifenbaum, M. Leder, and H. Reinhardt, Phys. Rev. D 73, 125019 (2006)

[23] W. Schleifenbaum, A. Maas, J. Wambach, and R. Alkofer, Phys. Rev. D 72, 014017 (2005).

[24] R. Alkofer, C. S. Fischer, and F. J. Llanes-Estrada, Phys. Lett. B 611, 279 (2005).

[25] A. Cucchieri, A. Maas, and T. Mendes, arXiv:hep-lat/0701011.

[26] A. Cucchieri, T. Mendes, and A. Mihara, JHEP 0412, 012 (2004).

[27] A. Cucchieri and T. Mendes, hep-lat/9902024.

[28] C. S. Fischer and D. Zwanziger, Phys. Rev. D 72, 054005 (2005).

[29] A. Cucchieri, A. Maas, T. Mendes, in preparation.

[30] "Finite-Temperature Field Theory", J. I. Kapusta (Cambridge University Press, 1989).

[31] A. Cucchieri and T. Mendes, hep-ph/0605224, and references therein.

[32] A. Cucchieri, F. Karsch, and P. Petreczky, Phys. Rev. D 64, 036001 (2001); Phys. Lett. B 497, 80 (2001).

[33] I. Zahed and D. Zwanziger, Phys. Rev. D 61, 037501 (2000); A. Maas, J. Wambach, and R. Alkofer, Eur. Phys. J. C 42, 93 (2005); A. Maas, Mod. Phys. Lett. A 20, 1797 (2005), and references therein. 